\title{
REDUCTIONS BETWEEN CERTAIN INCIDENCE PROBLEMS AND THE CONTINUUM HYPOTHESIS
}

\begin{abstract}
A b s t r a c t. In this work, we consider two families of incidence problems, $\mathcal{C}_{1}$ and $\mathcal{C}_{2}$, which are related to real numbers and countable subsets of the real line. Instances of problems of $\mathcal{C}_{1}$ are as follows: given a real number $x$, pick randomly a countable set of reals $A$ hoping that $x \in A$, whereas instances of problems of $\mathcal{C}_{2}$ are as follows: given a countable set of reals $A$, pick randomly a real number $x$ hoping that $x \notin A$. One could arguably defend that, at least intuitively, problems of $\mathcal{C}_{2}$ are easier to solve than problems of $\mathcal{C}_{1}$. After some suitable formalization, we prove (within $\mathbf{Z F C}$ ) that, on one hand, problems of $\mathcal{C}_{2}$ are, indeed, at least as easy to solve as problems of $\mathcal{C}_{1}$. On the other hand, the statement "Problems of $\mathcal{C}_{1}$ have the exact same complexity of problems of $\mathcal{C}_{2}$ " is shown to be an equivalent of the Continuum Hypothesis.
\end{abstract}

Received 18 February 2019

Keywords and phrases: Continuum Hypothesis, incidence problems, complexity, Dialectica Categories.

AMS subject classification: Primary 03E50; Secondary 18A05, 18 A15. 


\section{Introduction}

In what follows, we investigate some issues from the Set Theory of real numbers and we work within $\mathbf{Z F C}$ - that is, the Axiom of Choice (AC) and all of its equivalents are available. The presence of the Axiom of Choice is critical and carries some subtleties which will be addressed in the last section. We assume the reader is familiar with some intermediate set theory - including ordinals, cardinals and cofinalities; the reader may find all relevant definitions, notations and terminology in the standard reference [9]. The cardinality of a set $X$ is denoted by $|X|$. The family of all countably infinite subsets of $\mathbb{R}$ is denoted by $[\mathbb{R}]^{\aleph_{0}}$. The cardinality of the real numbers, $|\mathbb{R}|=2^{\aleph_{0}}$, is denoted by $\mathfrak{c}$ and is referred to as the cardinality of the continuum. $\mathbf{C H}$ denotes the Continuum Hypothesis, which is the statement " $c=\aleph_{1}$ " and is probably the most famous mathematical statement whose validity was proved to be independent of ZFC.

This paper will investigate some problems which may be viewed as thought experiments. In order to state such thought experiments, we have chosen to proceed with a certain ad hoc identification (in principle, by stylistic reasons; a thought experiment has to be compelling, otherwise it would be ignored - but, of course, there are no naive choices in science). We will identify randomness with arbitrariness. In practice, such identification consists in the assumption that, when we declare that we are picking arbitrary objects, then there is no pattern involved and all possible outcomes are unpredictable since they obey an equal probability distribution.

Let us turn to Theoretical Computer Science. In the field of Computational Complexity, one of the main techniques for relating and/or comparing the complexity of two problems is given by the notion of reduction. In the classical book of Garey and Johnson ([7]), a reduction of a problem $A$ to a problem $B$ is defined in the following way (see p.14, op.cit.): $A$ reduces to $B$ if there is a constructive transformation which maps any instance of $A$ into an equivalent instance of $B$; it should be clear that, in this context, "equivalent" means that a solution for the instance of $B$ which is obtained via transformation provides a solution for the original instance of $A$ (see [16], p. 159). One usually denotes " $A$ reduces to $B$ " by $A \leqslant B$; in such a case, it is usually said that " $B$ is at least as hard as $A$ ", or " $A$ is at least as easy as $B$ " (or also " $A$ is at least as simple to be solved as $B$ "). 
A mathematical counterpart of the notion of reduction in Computational Complexity is given by the morphisms of the category $\mathcal{P} \mathcal{V}$, which is a subcategory of the dual of the simplest case of the Dialectica Categories introduced by Valeria de Paiva ([13],[14]); such morphisms are also known as Galois-Tukey connections, which is a terminology due to Peter Vojtáš ([21]). Several connections between such category and Set Theory have been extensively studied by Andreas Blass in the 90's (see e.g. [3],[4]), and, more recently, have also been investigated by the author ([19],[20]).

$\mathcal{P} \mathcal{V}$ is the subcategory of the dual Dialectica category $\operatorname{Dial}_{2}(\text { Sets })^{\text {op }}$ whose objects - which, in general, are triples $(A, B, E)$, where $A, B$ and $E$ are sets and $E \subseteq A \times B$ - are those which satisfy the following conditions (which will be called the MHD conditions - where MHD stands for: Moore, Hrušák and Džamonja, see page 2283 of [11]):

(1) $0<|A|,|B| \leqslant 2^{\aleph_{0}}$.

(2) $(\forall a \in A)(\exists b \in B)[a E b]$

(3) $(\forall b \in B)(\exists a \in A)[\neg(a E b)]$

The morphisms between objects of $\mathcal{P} \mathcal{V}$ are the very same morphisms of $\operatorname{Dial}_{2}(\text { Sets })^{\text {op }}$ - that is, a morphism from an object $o_{2}=\left(A_{2}, B_{2}, E_{2}\right)$ to an object $o_{1}=\left(A_{1}, B_{1}, E_{1}\right)$ is a pair of functions $(\varphi, \psi)$, where $\varphi: A_{1} \rightarrow A_{2}$ and $\psi: B_{2} \rightarrow B_{1}$ are such that

$$
\left(\forall a \in A_{1}\right)\left(\forall b \in B_{2}\right)\left[\varphi(a) E_{2} b \longrightarrow a E_{1} \psi(b)\right]
$$

Morphisms of $\mathcal{P V}$ induce the so-called Galois-Tukey pre-order, which is defined in the following way: if $o_{1}=\left(A_{1}, B_{1}, E_{1}\right)$ and $o_{2}=\left(A_{2}, B_{2}, E_{2}\right)$ are objects of $\mathcal{P} \mathcal{V}$, then we have

$$
o_{1} \leqslant G T o_{2} \Longleftrightarrow \text { There is a morphism from } o_{2} \text { to } o_{1} \text {. }
$$

The diagram below represents the situation where $o_{1} \leqslant G T o_{2}$ :

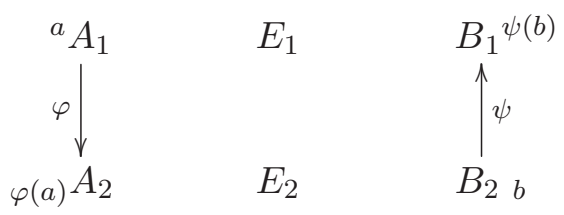


Given an object $o=(A, B, E)$ of $\mathcal{P} \mathcal{V}$, its dual object is given by $o^{*}=$ $\left(B, A, E^{*}\right)$, where $b E^{*} a$ means that $\neg a E b$. One can easily check (via a contrapositive argument) that:

$$
\text { If } o_{1} \leqslant G T o_{2} \text {, then } o_{2}^{*} \leqslant G T o_{1}^{*} \text {. }
$$

Blass interprets (see page 62 of [3]) each object $o=(A, B, E)$ in the following way: $O$ represents a certain problem (or a type of problem); $A$ is the particular set of instances of the problem represented by $o, B$ is the set of possible solutions of such problem and $E$ is the relation "is solved by", that is, $a E b$ says that " $b$ solves $a$ ".

In this sense, the Galois-Tukey pre-order indeed measures complexity: if $o_{1} \leqslant G T o_{2}$ then the problems in $o_{1}$ are not more complicated (that is, are at least as simple) to solve than problems in $o_{2}$, since every problem in $o_{1}$ may have its solution reduced to the solution of a problem in $o_{2}-$ or, more precisely, the act of solving a problem in $o_{1}$ may be reduced to the act of solving a (corresponding) problem in $o_{2}$. Indeed, under Blass' interpretation, the definition of the morphisms of $\mathcal{P} \mathcal{V}$ says that, in the case of $o_{1} \leqslant G T o_{2}$, if $b \in B_{2}$ is a solution for the instance $\varphi(a)$ of $o_{2}$ then $\psi(b) \in B_{1}$ is a solution for the instance $a$ of $o_{1} \cdot{ }^{1}$ These "GT-reductions" will play a key role in this work.

The described machinery of the $\mathcal{P} \mathcal{V}$ category will be applied for two families of incidence problems (which may also be regarded as challenges), $\mathcal{C}_{1}$ and $\mathcal{C}_{2}$, both related to real numbers and countably infinite subsets of the real line. In both cases, to solve the problem (or win the challenge) one has to give an appropriate (but random) response to a certain initial data; notice that such procedure could also be interpreted as some one-round game between two players, were the first player gives the initial data and the second player wins if he gives a response which solves the problem. Instances of problems of $\mathcal{C}_{1}$ are as follows: given a real number $x$, pick randomly a countably infinite set of reals $A$ hoping that $x \in A$, whereas

\footnotetext{
${ }^{1}$ Notice that the reductions also apply to the cases where one assumes that the input values are random variables. A function of a random variable is a random variable as well - see, e.g., [17], pages 121 and 208 -, thus, in the described context, if $a$ is a random instance of $A_{1}$ then $\varphi(a)$ may be regarded as a random instance of $A_{2}$, and the same argument applies, mutatis mutandis - that is, using $\psi$ - for a random instance $b$ of $B_{2}$ which solves $\varphi(a)$.
} 
instances of problems in $\mathcal{C}_{2}$ are as follows: given a countably infinite set of reals $A$, pick randomly a real number $x$ hoping that $x \notin A .^{2}$

Blass' interpretation of the triples of $\mathcal{P} \mathcal{V}$, described above, lead us to identify $\mathcal{C}_{1}$ with the object $\left(\mathbb{R},[\mathbb{R}]^{\aleph_{0}}, \in\right)$ : in this sense, each real number $x$ is an instance of the problem, whose possible solutions are countably infinite subsets $A \in[\mathbb{R}]^{\aleph_{0}}$ such that $x \in A$. And, in the precise same way, we identify $\mathcal{C}_{2}$ with the object $\left([\mathbb{R}]^{\aleph_{0}}, \mathbb{R}, \not \ni\right)$ : in this sense, each countably infinite subset $X$ is an instance of the problem, whose possible solutions are real numbers $x \in \mathbb{R}$ such that $x \notin A$.

One could arguably defend that, at least intuitively, problems of $\mathcal{C}_{2}$ are easier to solve than problems of $\mathcal{C}_{1}$ - since countable subsets of the real line have Lebesgue measure zero, and measure captures the notion of probability: if $A$ is countable, then $A$ is null and being a member of $A$ may be regarded as a very rare event, and so it should be (or, at least, one could plausibly believe that it should be) much more easy to pick randomly a real number which is not in $A$, rather than doing (essentially) the opposite.

The main aim of this research is to compare the complexities of $\mathcal{C}_{1}$ and $\mathcal{C}_{2}$, using $G T$-reductions to proceed with the measurements; such complexity comparisons relate, as will be shown, to both $\mathbf{C H}$ and $\mathbf{A C}$.

Let us describe the organization of this paper. In Section 2 the main results of the paper are established:

1. In the context of $G T$-reductions, we prove (within $\mathbf{Z F C}$ ) that, on one hand, problems of $\mathcal{C}_{2}$ are, indeed, at least as easy to solve as problems of $\mathcal{C}_{1}$.

2. On the other hand, the statement "Problems of $\mathcal{C}_{1}$ have the exact same complexity of problems of $\mathcal{C}_{2}$ " is shown to be an equivalent of the Continuum Hypothesis.

In Section 3, we show that the arguments presented in order to establish our equivalence for $\mathbf{C H}$ may be all formulated in terms of certain cardinal invariants of the ideal of countable subsets of the real line.

${ }^{2}$ In Section 4 we argue that, within a certain precise point of view, there is no difference between starting with the real number or with the countable subset - because, in fact, we will be able to assume that both of them will be given simultaneously. In Section 2 we will keep on the described formal statement of the problems, mostly because it fits perfectly with our main purpose, which is to investigate the action of $G T$-reductions. 
In the final section we present some notes and questions. A main question (regarding complexity and probability) emerges, and we debate whether the results of this paper provide information to the discussion of such question. In particular, we discuss certain relationships between the results of this paper and those from Freiling's famous paper on throwing darts at the real line $([5])$.

\section{Complexities and the Continuum Hypothesis}

Accordingly to the discussion at the Introduction, we proceed (via reductions) to the comparison of the complexities of the incidence problems given by $\mathcal{C}_{1}$ and $\mathcal{C}_{2}$. Throughout this section, $o_{1}$ and $o_{2}$ will refer to the following objects of $\mathcal{P} \mathcal{V}$ :

$$
o_{1}=\left(\mathbb{R},[\mathbb{R}]^{\aleph_{0}}, \in\right) \text {, and } o_{2}=\left([\mathbb{R}]^{\aleph_{0}}, \mathbb{R}, \not \supset\right) .
$$

Under our interpretation, $o_{1}$ represents the problems of $\mathcal{C}_{1}$ and $o_{2}$ represents the problems of $\mathcal{C}_{2}$ (as described in the Introduction).

First we prove that, under the described formalization, problems of $\mathcal{C}_{2}$ are, indeed, at least as simple to be solved as problems of $\mathcal{C}_{1}$.

Proposition 2.1. There is a morphism witnessing $o_{2} \leqslant_{G T} o_{1}$. In words: Problems of $\mathcal{C}_{2}$ are at least as simple to be solved as problems of $\mathcal{C}_{1}$.

Proof. The key of the proof is that, by the well-known König's Lemma, the cofinality of $2^{\aleph_{0}}$ is uncountable. Let us enumerate $\mathbb{R}=\left\{x_{\alpha}: \alpha<\mathfrak{c}\right\}$. For any $X \in[\mathbb{R}]^{\aleph_{0}}$, let $\gamma(X)<\mathfrak{c}$ be the ordinal

$$
\gamma(X)=\sup \left\{\alpha<\mathfrak{c}: x_{\alpha} \in X\right\}+1 \text {. }
$$

Let $\varphi:[\mathbb{R}]^{\aleph_{0}} \rightarrow \mathbb{R}$ be defined by putting, for every $X \in[\mathbb{R}]^{\aleph_{0}}$,

$$
\varphi(X)=x_{\gamma(X)} .
$$

Notice that, for any $X \in[\mathbb{R}]^{\aleph_{0}}$, every element of $X$ has its ordinal index strictly smaller than the ordinal index of $\varphi(X)$. 
Let us consider $\psi=\varphi$, that is, we take the pair of functions given by $(\varphi, \varphi)$ as a candidate to be a morphism. We claim that such pair is, in fact, a morphism of $\mathcal{P} \mathcal{V}$, from $o_{1}$ to $o_{2}$.

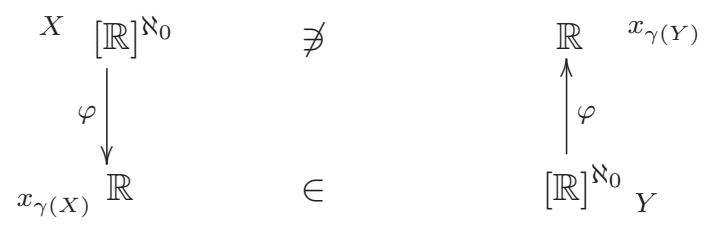

Indeed: let $X, Y$ be any countably infinite subsets of $\mathbb{R}$. If $\varphi(X)=$ $x_{\gamma(X)} \in Y$, then $\gamma(X)<\gamma(Y)$, and therefore $x_{\gamma(Y)}=\varphi(Y) \notin X$.

As announced, whether the reverse $G T$-inequality between $o_{1}$ and $o_{2}$ holds or not is a question whose answer is independent of ZFC.

Theorem 2.2. The following statements are equivalent:

(i) The Continuum Hypothesis.

(ii) $o_{1} \leqslant G T o_{2}$.

In words: Problems of $\mathcal{C}_{1}$ are at least as simple to be solved as problems of $\mathcal{C}_{2}$.

(iii) $o_{1} \cong_{G T} O_{2}$.

In words: Problems of $\mathcal{C}_{1}$ have the exact same complexity of problems of $\mathcal{C}_{2}$.

Proof. As we have proved that $o_{2} \leqslant G T o_{1}$ holds in ZFC, the equivalence between (ii) and (iii) is clear; so, it suffices to prove the equivalence between $(i)$ and $(i i)$.

Proof of $(i) \Rightarrow($ ii): Assuming $\mathbf{C H}$, we are allowed to enumerate the reals as $\mathbb{R}=\left\{x_{\alpha}: \alpha<\omega_{1}\right\}$. Let $\varphi: \mathbb{R} \rightarrow[\mathbb{R}]^{\aleph_{0}}$ be the function defined in the following way: for every $\alpha<\omega_{1}$,

$$
\varphi\left(x_{\alpha}\right)=\left\{\begin{aligned}
\left\{x_{\xi}: \xi \leqslant \alpha\right\} & \text { if } \alpha \geqslant \omega ; \text { and } \\
\left\{x_{n}: n<\omega\right\} & \text { otherwise. }
\end{aligned}\right.
$$


Notice that the reals indexed by finite ordinals (i.e., indexed by natural numbers) are elements of $\varphi(x)$ for any real number $x$.

Let us consider $\psi=\varphi$, that is, again we take a pair of functions of functions of the form $(\varphi, \varphi)$ as a candidate to be a morphism. We claim that such pair is, indeed, a morphism of $\mathcal{P} \mathcal{V}$, from $o_{2}$ to $o_{1}$.

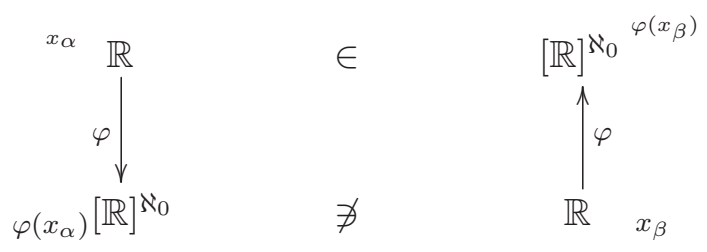

Indeed: let $x=x_{\alpha}$ and $y=x_{\beta}$ be arbitrary real numbers. If $x_{\beta} \notin \varphi\left(x_{\alpha}\right)$ then $\beta$ is an infinite ordinal, and also one has, necessarily, $\beta>\alpha$. It follows that $x_{\alpha} \in \varphi\left(x_{\beta}\right)$. This shows that $(\varphi, \varphi)$ is a morphism of $\mathcal{P} \mathcal{V}$ which witnesses $o_{1} \leqslant G T O_{2}$, as desired.

Proof of $($ ii $) \Rightarrow(i)$ : We argue contrapositively: assuming $\neg \mathbf{C H}$, we show that no pair of functions $(\varphi, \psi)$ is a morphism from $o_{2}$ to $o_{1}$. Thus, we have to check that, under $2^{\aleph_{0}}>\aleph_{1}$, the following formula holds:

$$
\left(\forall \varphi, \psi: \mathbb{R} \rightarrow[\mathbb{R}]^{\aleph_{0}}\right)(\exists x, y \in \mathbb{R})[\neg(y \notin \varphi(x) \rightarrow x \in \psi(y))],
$$

or, equivalently,

$$
\left(\forall \varphi, \psi: \mathbb{R} \rightarrow[\mathbb{R}]^{\aleph_{0}}\right)(\exists x, y \in \mathbb{R})[y \notin \varphi(x) \wedge x \notin \psi(y)] .
$$

Fix $A \subseteq \mathbb{R}$ with $|A|=\aleph_{1}$. It follows that

$$
\left|\bigcup_{x \in A} \varphi(x)\right| \leqslant \aleph_{1}
$$

and, as we are assuming $2^{\aleph_{0}}>\aleph_{1}$, we may pick some $y \notin \bigcup_{x \in A} \varphi(x)$. To get done, notice that, as $\psi(y)$ is a countable subset of the reals, we necessarily have $A \backslash \psi(y) \neq \emptyset$ - and therefore we may pick some $x \in A$ such that $x \notin \psi(y)$. For this particular pair $\{x, y\}$ of reals we have what we want, that is, $y \notin \varphi(x)$ and $x \notin \psi(y)$. 


\section{Combinatorics of Ideals}

It is possible to reformulate our main result (namely, the equivalence between $\mathbf{C H}$ and the statement " $\left.\left(\mathbb{R},[\mathbb{R}]^{\aleph_{0}}, \in\right) \cong_{G T}\left([\mathbb{R}]^{\aleph_{0}}, \mathbb{R}, \not \supset\right) "\right)$ in the language of combinatorics of ideals. As it is probably known to the reader, a family $\mathcal{I}$ of subsets of a non-empty set $X$ is said to be an ideal of subsets of $X$ if it is a proper, non-empty subset of $\mathcal{P}(X)$ which is closed under taking subsets and under taking finite unions.

In all of our arguments, one could easily replace

$$
\left(\mathbb{R},[\mathbb{R}]^{\aleph_{0}}, \in\right) \text { and its dual object }\left([\mathbb{R}]^{\aleph_{0}}, \mathbb{R}, \not \supset\right)
$$

by the objects

$$
\left(\mathbb{R},[\mathbb{R}]^{\leqslant \aleph_{0}}, \in\right) \text { and its dual object }\left([\mathbb{R}]^{\leqslant \aleph_{0}}, \mathbb{R}, \not \nexists\right),
$$

where $[\mathbb{R}]^{\leqslant \aleph_{0}}=\left\{Y \subseteq \mathbb{R}:|Y| \leqslant \aleph_{0}\right\}$ - that is, $[\mathbb{R}]^{\leqslant \aleph_{0}}$ is the family of all countable subsets of $\mathbb{R}$, including the finite ones. With such change, we get that $[\mathbb{R}]^{\leqslant \aleph_{0}}$ is an ideal of subsets of $\mathbb{R}$ - in fact, a $\sigma$-ideal (i.e., closed under countably infinite unions) which includes all singletons. There are certain cardinal invariants which can be associated, in general, to any ideal, but their behaviour is quite well-known in the case where the ideal is a $\sigma$-ideal including all the singletons.

Definition 3.1. (Cardinal invariants related to ideals).

Let $\mathcal{I}$ be an ideal of subsets of an infinite set $X$.

(i) $\operatorname{add}(\mathcal{I})$ (the additivity of $\mathcal{I}$ ) is the smallest size of a subfamily of $\mathcal{I}$ whose union is not in $\mathcal{I}$ - that is,

$$
\operatorname{add}(\mathcal{I})=\min \{|\mathcal{A}|: \mathcal{A} \subseteq \mathcal{I} \text { and } \bigcup \mathcal{A} \notin \mathcal{I}\} .
$$

(ii) $\operatorname{cov}(\mathcal{I})$ (the covering number of $\mathcal{I}$ ) is the smallest size of a subfamily of $\mathcal{I}$ which covers $X$ - that is,

$$
\operatorname{cov}(\mathcal{I})=\min \{|\mathcal{A}|: \mathcal{A} \subseteq \mathcal{I} \text { and } \bigcup \mathcal{A}=X\}
$$

(iii) $\operatorname{non}(\mathcal{I})$ (the uniformity of $\mathcal{I}$ ) is the smallest size of a subset of $X$ which is not in $\mathcal{I}$ - that is,

$$
\operatorname{non}(\mathcal{I})=\min \{|A|: A \subseteq X \text { and } A \notin \mathcal{I}\} .
$$


(iv) $\operatorname{cof}(\mathcal{I})$ (the cofinality of $\mathcal{I}$ ) is the smallest size of a subfamily of $\mathcal{I}$ which is cofinal in $\mathcal{I}$ - that is,

$$
\operatorname{cof}(\mathcal{I})=\min \{|\mathcal{A}|: \mathcal{A} \subseteq \mathcal{I} \text { and }(\forall I \in \mathcal{I})(\exists A \in \mathcal{A})[I \subseteq A]\} .
$$

It is easy to check that, for a $\sigma$-ideal $\mathcal{I}$ which includes all the singletons, the following inequalities hold:

$$
\begin{aligned}
\aleph_{1} \leqslant \operatorname{add}(\mathcal{I}) & \leqslant \min \{\operatorname{cov}(\mathcal{I}), \operatorname{non}(\mathcal{I})\} \\
& \leqslant \max \{\operatorname{cov}(\mathcal{I}), \operatorname{non}(\mathcal{I})\} \leqslant \operatorname{cof}(\mathcal{I}) \leqslant|\mathcal{I}| .
\end{aligned}
$$

In the cases where $\mathcal{I}$ is an ideal of subsets of $\mathbb{R}$, those cardinals invariants are examples of the so-called norms (or evaluations) of objects of $\operatorname{Dial}_{2}\left(\right.$ Sets) ${ }^{\text {op }}$ which satisfy the MHD conditions (2) and (3); for this work, we may assume that all objects are, in fact, in $\mathcal{P} \mathcal{V} .^{3}$

Definition 3.2. (Norms of $\mathcal{P V}$ objects).

Let $o=(A, B, E)$ be an object of $\mathcal{P} \mathcal{V}$. Its norm is the cardinal number $\|o\|=\|(A, B, E)\|$ given by

$$
\|o\|=\min \{|Y|: Y \subseteq B \text { and }(\forall a \in A)(\exists b \in Y)[a E b]\} .
$$

One can easily check that, indeed, MHD conditions (2) and (3) ensure that for any object $o \in \operatorname{obj}(\mathcal{P V})$ both $o$ and $o^{*}$ have well defined norms.

The application of $\mathcal{P} \mathcal{V}$ to Set Theory which was extensively studied by Blass in the 90's (see e.g. [3],[4]), and, more recently, by the author ([19],[6],[20]), is the so-called method of morphisms in the proof of inequalities between cardinal invariants of the continuum - which was once declared by Blass as being an empirical fact.

${ }^{3}$ The ideal $\mathcal{I}$ has to satisfy $|\mathcal{I}| \leqslant 2^{\aleph_{0}}$ in order to certain objects (which will be presently described) be, formally, objects of $\mathcal{P} \mathcal{V}$; notice that this is the case for the ideal of all countable subsets of $\mathbb{R}$. However, it is worthwhile mentioning that for the ideals $\mathcal{M}$ of all meager subsets of $\mathbb{R}$ and $\mathcal{L}$ of all null subsets of $\mathbb{R}$ (which both have size $2^{2^{\aleph_{0}}}$ ), the corresponding objects are considered as being objects of $\mathcal{P} \mathcal{V}$ within the literature, and this is justified by the fact that each of those two ideals have a basis of Borel sets; recall that there are exactly $2^{\aleph_{0}}$ Borel subsets of $\mathbb{R}$. 
It is an empirical fact that proofs of inequalities between cardinal characteristics of the continuum usually proceed by representing the characteristics as norms of objects in $\mathcal{P} \mathcal{V}$ and then exhibiting explicit morphisms between those objects (A. Blass, 1995 [3]).

The influence of the morphisms (and of the $G T$-ordering) in this context is due to the following result - which is the method of morphisms, so to say.

Theorem 3.3. ("Folklore"; cited in [4]). Let $o_{1}$ and $o_{2}$ be objects of $\mathcal{P V}$. If $o_{1} \leqslant G T o_{2}$ then $\left\|o_{1}\right\| \leqslant\left\|o_{2}\right\|$.

Thus, clearly one has that if $o_{1} \cong_{G T} o_{2}$ then $\left\|o_{1}\right\|=\left\|o_{2}\right\|$.

The easy proof of the above theorem is left as an exercise to the reader (but may be found in [6]). Certain relationships between the method of morphisms and the Axiom of Choice were investigated in [20].

And, as we have already mentioned, if $o_{1} \leqslant G T o_{2}$ then $o_{2}^{*} \leqslant G T o_{1}^{*}-$ so, in fact, when one shows $o_{1} \leqslant G T o_{2}$ both inequalities $\left\|o_{1}\right\| \leqslant\left\|o_{2}\right\|$ and $\left\|o_{2}^{*}\right\| \leqslant\left\|o_{1}^{*}\right\|$ are established.

Having the method of morphisms available, now we may explain how to reformulate our main result in the language of the combinatorics of ideals. First, we kindly invite the reader to realize that, in fact, the cardinal invariants defined for ideals are norms of objects: more precisely, given an ideal $\mathcal{I}$, one has that

$$
\begin{aligned}
\operatorname{add}(\mathcal{I}) & =\|(\mathcal{I}, \mathcal{I}, \nsupseteq)\|, \\
\operatorname{non}(\mathcal{I}) & =\|(\mathcal{I}, X, \nexists)\|, \\
\operatorname{cov}(\mathcal{I}) & =\|(X, \mathcal{I}, \in)\|, \\
\operatorname{cof}(\mathcal{I}) & =\|(\mathcal{I}, \mathcal{I}, \subseteq)\| .
\end{aligned}
$$

It follows that, letting $\mathcal{I}_{C}=[\mathbb{R}]^{\leqslant \aleph_{0}}$ denote the ideal of all countable subsets of $\mathbb{R}$, then the equivalence

$$
\mathbf{C H} \Longleftrightarrow\left(\mathbb{R}, \mathcal{I}_{C}, \in\right) \cong_{G T}\left(\mathcal{I}_{C}, \mathbb{R}, \not\right)
$$

implies (by the method of morphisms) the following statement: "If $\mathbf{C H}$ holds, then $\left\|\left(\mathbb{R}, \mathcal{I}_{C}, \in\right)\right\|=\left\|\left(\mathcal{I}_{C}, \mathbb{R}, \not ̋\right)\right\| "$ - that is, 


$$
\mathbf{C H} \Rightarrow \operatorname{cov}\left(\mathcal{I}_{C}\right)=\operatorname{non}\left(\mathcal{I}_{C}\right)
$$

However, it is easily seen (as an absolute, $\mathbf{Z F C}$ result) that the covering number of $\mathcal{I}_{C}$ is $\mathfrak{c}$ (since if $\lambda<\mathfrak{c}$ then any union of $\lambda$ countable sets has size not larger than $\max \left\{\lambda, \aleph_{0}\right\}<\mathfrak{c}$ ), and, even more clearly, the uniformity of $\left(\mathcal{I}_{C}\right)$ is $\aleph_{1}$. With these absolute, $\mathbf{Z F C}$ values for $\operatorname{cov}\left(\mathcal{I}_{C}\right)$ and $\operatorname{non}\left(\mathcal{I}_{C}\right)$ in mind, the arguments (via morphisms) presented for our equivalence of $\mathbf{C H}$, when regarded as proofs in the context of cardinal invariants of ideals, reduce the equivalence to a quite compact formula:

$$
\mathbf{C H} \Longleftrightarrow \underbrace{\operatorname{cov}\left(\mathcal{I}_{C}\right)}_{\mathfrak{c}}=\underbrace{\operatorname{non}\left(\mathcal{I}_{C}\right)}_{\aleph_{1}}
$$

\section{Notes and Questions}

We have decided to present the problems of $\mathcal{C}_{1}$ and $\mathcal{C}_{2}$ as incidence problems in order to emphasize the geometric appealing of those questions. However, it is thought-provoking to notice that they also could have been presented as decision problems. According to Garey and Johnson ([7], page 18), a decision problem is a problem which has only two possible solutions, "yes" or "no", meaning that there is some yes-or-no question (posed in term of the input values) such that the problem is to determine the correct answer to such question. Formally, a decision problem $\Pi$ consists of a set $D_{\Pi}$ of instances, and a subset $Y_{\Pi} \subseteq D_{\Pi}$ of yes-instances. Within this modelling, a decision problem $\Pi_{1}(A)$ of $\mathcal{C}_{1}$ has the following structure:

$$
\begin{aligned}
& D_{\Pi_{1}(A)}=\mathbb{R} \\
& Y_{\Pi_{1}(A)}=A \\
& \text { INPUT: } \quad x
\end{aligned}
$$

Y-N QUESTION: Is it true that $x \in A$ ?

A decision problem $\Pi_{2}(A)$ of $\mathcal{C}_{2}$ has the following structure:

$$
\begin{aligned}
& D_{\Pi_{2}(A)}=\mathbb{R} \\
& Y_{\Pi_{2}(A)}=\mathbb{R} \backslash A
\end{aligned}
$$

INPUT: $x$ 
Y-N QUESTION: Is it true that $x \notin A$ ?

Problems in $\mathcal{C}_{2}$ are, in fact, the complements of the problems of $\mathcal{C}_{1}$; more precisely, $\Pi_{2}(A)=\Pi_{1}(\mathbb{R} \backslash A)=\left(\Pi_{1}(A)\right)^{c}$. The complement of a decision problem $\Pi$ (denoted by $\Pi^{c}$ ) is, by definition, the decision problem whose answer is "yes" whenever the input is a "no" input of $\Pi$, and vice-versa ([16], page 142).

Notice also that, within this presentation, we are assuming that $x$ and $A$ may be given simultaneously, either for the problems of $\mathcal{C}_{1}$ or for the problems of $\mathcal{C}_{2}$ - that is, given an unordered pair $\{A, x\}$, picked arbitrarily, one can consider either the decision problem $\Pi_{1}(A)$ with input $x$ or the decision problem $\Pi_{2}(A)$ with input $x$, depending on the case we are interested in.

In almost purely layman's terms, we have turned the problems of $\mathcal{C}_{1}$ and $\mathcal{C}_{2}$ into the following (where $x$ and $A$ are as expected):

Problems of $\mathcal{C}_{1}$ (Answering YES to the corresponding decision problem):

To randomly pick - simultaneously or in any sequential order, it does not matter $-x$ and $A$ such that $x \in A$.

Problems of $\mathcal{C}_{2}$ (Answering YES to the corresponding decision problem):

To randomly pick - simultaneously or in any sequential order, it does not matter $-x$ and $A$ such that $x \notin A$.

Of course, this simplification explicitly presupposes that there is no difference between start considering the real number or start considering the countably infinite subset of the reals (in both families of problems, $\mathcal{C}_{1}$ and $\mathcal{C}_{2}$ ); both possibilities are allowed, together with the possibility of simultaneity.

This assumption of the possibility of simultaneity is critical, so let us elaborate some more on it. As we have remarked in the Introduction, we are identifying the notions of arbitrariness and randomness; this was an ad hoc identification, which was done, mostly, for the stylistic purposes of presentation of the thought experiments of this paper - but let us use a little bit of such identification in an argument, just once. Identifying arbitrariness and randomness, the beginning of our two thought experiments could be described in the same way: we may consider that we are able to pick (randomly) one element of each of, say, two jars: one of the jars contains 
all real numbers, and the other one contains all countably infinite subsets of the real line. And, in the same way that tossing a coin 2 times, sequentially, is equivalent, in terms of probability, to tossing 2 coins simultaneously, we may assume that our arbitrary pick of a real number and of a countable subset of the reals (to solve a problem of $\mathcal{C}_{1}$ or of $\mathcal{C}_{2}$, depending on the case) may be done simultaneously, since "being a certain real number" and "being a certain countably infinite subset of the reals" are, clearly, independent events. And, as the order of those events does not matter, as we can even assume that they are simultaneous, we are also able to avoid, if we want to, further discussions regarding the notion of symmetry; at most, we could recognize that, in our context, symmetry may be regarded as a merely, simple consequence of the independence we have just referred to.

Also, it is clear that one is able to drop the terminology of "morphisms between objects of a certain category" and work directly with the families of decision problems given by $\mathcal{C}_{1}$ and $\mathcal{C}_{2}$ to get to the very same conclusions, via reductions (decision problems of $\mathcal{C}_{2}$ are at least as simple to be solved as problems of $\mathcal{C}_{1}$, and the reciprocal statement is equivalent to $\mathbf{C H}$ ).

All things considered (regarding the described modelling of $\mathcal{C}_{1}$ and $\mathcal{C}_{2}$ as families of decision problems), it seems that the results of this paper provide information for the discussion of the following question:

Question 4.1. (The Main Question). Before being given a countable set $A$ of reals and a real number $x$, both to be randomly taken, should one say that it will be easier (or it will be more likely) that, eventually, this real number $x$ will miss the countable set $A$ ? Or should one say that, under the very same conditions and interpretations, it will hit it?

Notice that, in the preceding question, "being easier" refers to complexity, and "being more likely" refers to probability. The results of this paper show that, if one accepts the exhibiting-reductions approach to discuss the complexity of the problems $\mathcal{C}_{1}$ and $\mathcal{C}_{2}$, then a full answer to the "complexity part" of the Main Question depends on the Continuum Hypothesis and therefore we would have faced an undecidable problem (in the sense that the statement "Problems of $\mathcal{C}_{1}$ have the exact same complexity of problems of $\mathcal{C}_{2}$ " was shown to be independent of the usual axioms of Set Theory).

In natural sciences, complexity and probability are usually regarded as related notions. It is largely accepted in those fields that, when it comes to 
natural systems, "the more complex, the less probable".

From the perspective of the physical sciences, explaining life is a highly challenging task because the more complex a system is, the less probable it becomes both in its appearance and its persistence (A. Moreno and M. Mossio, 2015 [12]).

The results of this paper presuppose background and terminology of computational complexity, and, of course, we cannot assume that there is a direct, straightforward link between the notions of complexity of natural systems and computational complexity; so, we are not in position to embrace here in this paper some version of the slogan "the more complex, the less probable". Nevertheless, it is quite interesting to point out that ZFC was able to establish $o_{2} \leqslant_{G T} o_{1}$, which states that, accordingly to our probability intuition, problems of $\mathcal{C}_{1}$ are more complex than problems of $\mathcal{C}_{2}$. It is also worth mentioning that, in the Philosophy of Science, it has been recognized that the possibility of simulation of computational models of complex systems, as well as the technique of encapsulation (that is, to divide a large, complex problem into smaller and simpler ones), have been constituted themselves into important tools in the study of complexity within science.

One of the greatest effects of modern computing on science had been to make it possible to attack complex questions that previously were intractable. By making simulation modelling practical, computers have helped to advance complexity theory in several ways (David G. Green and Tania Leishman, 2011 [8]).

Also regarding the "probability part" of the Main Question, it is engaging to proceed some comparisons with a previous work of Freiling. Indeed, the (proof of the) equivalence of $\mathbf{C H}$ presented in this paper is quite similar to one among a number of equivalences presented by Freiling in [5]. The main point of Freiling, however, was to present a "philosophical proof" of the negation of the Continuum Hypothesis (as explicitly declared in the very first phrase of the abstract of [5]), based on certain probability reasonings. In fact, the mathematical content of Freiling's proof is due to Sierpiński and was presented in the third decade of XX century, in his classical monograph on the Continuum Hypothesis ([18]). 
Freiling's argument is exposed in terms of a thought experiment involving darts. In an elegant (but, of course, debatable) appeal to intuition, his paper suggests, in fact, a number of thought experiments, so that a countably infinite subset of $\mathbb{R}$ may be regarded, ultimately, as being the set of punctured points obtained by throwing a countably infinite set of darts at the real line.

As the title itself of Freiling's article indicates, a main part of his argument against $\mathbf{C H}$ is to assume that there is a symmetry in a certain situation. Next, we expose a slight variation of Freiling's symmetry argument using the language of games; such variation is due to Aurichi and was presented in his MSc Dissertation ([1]).

Suppose that for each real number $x$ we have associated a countably infinite set of reals, $A_{x}$ - that is, we are considering a certain function $f: \mathbb{R} \rightarrow[\mathbb{R}]^{\aleph_{0}}$ which represents a given, random association $x \mapsto A_{x}$ (so that $A_{x}=f(x)$ ); to start to stick with the whole darts idea, we could think that a randomly taken countably infinite subset of $\mathbb{R}$ is the set of punctured points obtained by a certain random throw of a countably infinite set of darts at the real line, and, accordingly, we may think that, in our context, every real number $x$ has thrown countable darts which have hit the real line precisely at the points of the assigned countable set $A_{x}$. Two players, ONE and TWO, will play a one-round game, as follows: ONE will throw a dart at the real line, hitting a real number $x_{1}$. After that, TWO will throw a dart at the real line, hitting a real number $x_{2}$; notice that these last two dart throws resemble the fundamental assumption that $x_{1}$ and $x_{2}$ were randomly selected. ONE wins the game if $x_{1} \in A_{x_{2}}$ or $x_{2} \in A_{x_{1}}$, otherwise TWO wins.

Now, assume that ONE and TWO have played their darts, so we may consider real numbers $x_{1}$ and $x_{2}$ as above; let us give a blindfolded prediction on the outcome of this game. We have that $A_{x_{1}}$ is a countable set, and therefore has null measure - and thus one could state (or predict) that it is much more likely that $x_{2} \notin A_{x_{1}}$ (indeed, with probability 1 ), and (here comes the argument of symmetry) as the real number line does not really know which dart was thrown first or second (those were precisely Freiling's words), also with probability 1 one could state that, in fact, it is much more likely that both " $x_{1} \notin A_{x_{2}}$ " and " $x_{2} \notin A_{x_{1}}$ " hold; that is, even blindfolded we are able to declare that it is almost a sure thing that TWO has won the game. Freiling also observes that, if in this very first play between 
ONE and TWO, "by some very strange miracle", it turned out to happen (say) $x_{2} \in A_{x_{1}}$, then we could always ask ONE and TWO to throw the darts again, concluding that "what heuristically will happen any time, can happen" - meaning, the probability of getting $x_{1} \notin A_{x_{2}}$ and $x_{2} \notin A_{x_{1}}$ is so high that such outcome would be surely obtained after a few throws of darts. ${ }^{4}$

With such arguments (delivered by Freiling as a "philosophical justification"), one could conclude that the following formula should be valid:

$$
\mathbf{A}_{\aleph_{0}} \equiv\left(\forall f: \mathbb{R} \rightarrow[\mathbb{R}]^{\aleph_{0}}\right)(\exists x, y \in \mathbb{R})[y \notin f(x) \wedge x \notin f(y)] .
$$

And finally, using an argument very similar to our proof of the equivalence $(i) \Longleftrightarrow($ ii $)$ of Theorem 2.2, Freiling has shown that

$$
\mathbf{A}_{\aleph_{0}} \Longleftrightarrow \neg \mathbf{C H}
$$

We believe that our approach has some advantages, in the comparison with the one of Freiling's, mainly by three reasons:

1. Freiling has never formalized his probability argument; his philosophical justification was only intuitive. In fact, the only formal treatment (using Probability Theory) of the darts problem the author is aware of is the one presented by Aurichi in the Section 3.6 of [1].

2. After his intuitive argument, Freiling came to his formula $\mathbf{A}_{\aleph_{0}}$ and has shown its equivalence with $\neg \mathbf{C H}$. In our work, a very similar formula, which is

$$
\left(\forall \varphi, \psi: \mathbb{R} \rightarrow[\mathbb{R}]^{\aleph_{0}}\right)(\exists x, y \in \mathbb{R})[y \notin \varphi(x) \wedge x \notin \psi(y)]
$$

has naturally appeared under a formal and well-defined mathematical context: the one of comparing the complexities of the problems of $\mathcal{C}_{1}$ and $\mathcal{C}_{2}$. Such meaningful formula (whose validity was shown to be a consequence of $\neg \mathbf{C H}$ in $\mathbf{Z F C}$ ) allowed us to establish the following statement: if the Continuum Hypothesis is false, then there are no

\footnotetext{
${ }^{4}$ In fact, one could wonder if Freiling's argument isn't actually presupposing that "what heuristically will happen any time, will happen some time".
} 
possible reductions of problems of $\mathcal{C}_{1}$ to problems of $\mathcal{C}_{2}$. So, we believe the results of this paper, which constitute formal theorems on their own, were stated under a very specified, clear and motivated mathematical context.

3. We have presented here a formulation on which there is no need of discussing symmetry issues (namely, the modelling of $\mathcal{C}_{1}$ and $\mathcal{C}_{2}$ as families of decision problems). In fact, there are no arguments of symmetry in the proofs of this paper. Nevertheless, recall we have made quite clear that our modelling of $\mathcal{C}_{1}$ and $\mathcal{C}_{2}$ as families of decision problems embrace (by presuposing) concepts as independence and simultaneity.

As we come close to finishing this work, we have to admit that a number of objections which have been made to Freiling's darts could also apply to our reductions; for instance, one could criticize and/or question the role of the Axiom of Choice in all what have been done. As pointed out by Maddy in [10], natural generalizations of Freiling's statement $\mathbf{A}_{\aleph_{0}}$ contradict $\mathbf{A C}$ as well - that is, Freiling's case against $\mathbf{C H}$ may be, in fact, a case against AC. For instance, if one considers that "being a member of a set whose cardinality is less than $\mathfrak{c}$ " is also a very rare event, then one could proceed with a similar "darts game argument" to conclude that the following formula should be valid:

$$
\mathbf{A}_{<\mathfrak{c}} \equiv\left(\forall f: \mathbb{R} \rightarrow[\mathbb{R}]^{<\mathfrak{c}}\right)(\exists x, y \in \mathbb{R})[y \notin f(x) \wedge x \notin f(y)]
$$

Nonetheless, it is easily seen that the statement " $\mathbb{R}$ can be well ordered" implies that $\mathbf{A}_{<\mathfrak{c}}$ is not valid (one has just pick some well ordering of $\mathbb{R}$ with order type $\mathfrak{c}$, say $\langle\mathbb{R}, \prec\rangle$, and then the function $f: \mathbb{R} \rightarrow[\mathbb{R}]^{<\mathfrak{c}}$ given by $f(x)=\{y: y \preceq x\}$ is a counterexample to $\left.\mathbf{A}_{<\mathfrak{c}}\right)$. It follows that $\mathbf{A}_{<\mathfrak{c}}$ is inconsistent with ZFC.

Working similarly as in this paper, one could design problems which, under the $\mathcal{P} \mathcal{V}$ interpretations, could be represented by $\left(\mathbb{R},[\mathbb{R}]^{<\mathfrak{c}}, \in\right)$ and $\left([\mathbb{R}]^{<\mathfrak{c}}, \mathbb{R}, \not \supset\right)$, in such a way that $\left(\mathbb{R},[\mathbb{R}]^{<\mathfrak{c}}, \in\right) \leqslant G T\left([\mathbb{R}]^{<\mathfrak{c}}, \mathbb{R}, \not \supset\right)$ follows, in $\mathbf{Z F}$, from a well-ordering of $\mathbb{R}$. Regarding the absolute result of Proposition 2.1 (i.e., ZFC proves $o_{2} \leqslant G T o_{1}$ ), if one tries to adapt its proof in order to establish $\left([\mathbb{R}]^{<\mathfrak{c}}, \mathbb{R}, \not \supset\right) \leqslant_{G T}\left(\mathbb{R},[\mathbb{R}]^{<\mathfrak{c}}, \in\right)$, then the easy conclusion is that, in fact, such $G T$-inequality follows, within $\mathbf{Z F C}$, from the statement " $2{ }^{\aleph_{0}}$ 
is a regular cardinal" (which holds, for instance, under Martin's Axiom see [9], page 58).

The preceding paragraph justifies the following problem:

Problem 4.2. Determine the precise deductive strengths of

(i) $\left(\mathbb{R},[\mathbb{R}]^{<\mathfrak{c}}, \in\right) \leqslant G T\left([\mathbb{R}]^{<\mathfrak{c}}, \mathbb{R}, \not \supset\right)$, relatively to $\mathbf{Z F}$; and

(ii) $\left([\mathbb{R}]^{<\mathfrak{c}}, \mathbb{R}, \not\right) \leqslant_{G T}\left(\mathbb{R},[\mathbb{R}]^{<\mathfrak{c}}, \in\right)$, relatively to $\mathbf{Z F C}$.

Even the absolute ZFC result we have just mentioned - Proposition 2.1, $o_{2} \leqslant G T o_{1}$ - encompasses critical uses of the Axiom of Choice. First of all, and more obviously, the function $\varphi$ defined in its proof is given in terms of an enumeration (essentially, a well-ordering) of $\mathbb{R}$. Second, and less obviously, one should recall that König's Lemma - which says that for all infinite cardinal $\kappa$, the cofinality of $2^{\kappa}$ is larger than $\kappa$ - is a corollary of the so-called König's Theorem; and such theorem may be rephrased to the following $\mathbf{Z F}$ statement, which in turn is easily seen to be an equivalent of the Axiom of Choice:

"If $I$ is a non-empty set and $\left\{A_{i}: i \in I\right\}$ and $\left\{B_{i}: i \in I\right\}$ are families of sets such that, for all $i \in I, A_{i}$ is strictly dominated by $B_{i}$ for every $i \in I$, then $\bigcup_{i \in I}\left(A_{i} \times\{i\}\right)$ is strictly dominated by $\prod_{i \in I} B_{i}$."

The preceding considerations on the role of the Axiom of Choice in this work justifies the following question.

Question 4.3. What happens if we consider the families of problems $\mathcal{C}_{1}$ and $\mathcal{C}_{2}$ in a choiceless Set Theory? What is the precise role of the Axiom of Choice in all results of this paper? How much of the Axiom of Choice is needed in order to get to the very same conclusions?

A question (raised by the referee) is whether the so-called Chu constructions (or Chu transforms), proposed by Barr and Chu in [2] and used in Category Theory and Computer Science, could turn out to be a kind of alternative way to express some of the arguments of this paper. As pointed out by Blass ([3]) and de Paiva ([15]), there are similarities and differences between $\mathcal{P} \mathcal{V}$ and Chu constructions.

Linear Logic has been much investigated using categorical methods. In particular two generic constructions, the Chu construction and the 
Dialectica construction were used to provide general ways of building classes of (categorical) models for Linear Logic. The construction themselves are similar in many ways, but different in significant others, so it is difficult to compare them abstractly (Valeria de Paiva, $2006[15])$.

There are strong but superficial similarities between $\mathcal{P} \mathcal{V}$ and a special case of a construction due to Chu... Specifically, Chu's construction, applied to the cartesian closed category of sets and the object 2, yields a $*$-autonomous category in which the objects are the same as those of $\mathcal{P V}$ and the morphisms differ from those of $\mathcal{P} \mathcal{V}$ only in that they are required to satisfy... (an equivalence) rather than just an implication from left to right. This apparently minor difference in the definition leads to major differences in other aspects of the category, specifically in the internal hom-functor and the tensor product (Andreas Blass, $1995[3])$.

It is also interesting to notice that such a requirement of an equivalence as, say,

$$
\varphi(a) E_{2} b \longleftrightarrow a E_{1} \psi(b)
$$

in the definition of the morphisms in Chu categories also corresponds to a different notion of reduction between problems: under this notion, and considering the families of problems given by the objects $o_{1}=\left(A_{1}, B_{1}, E_{1}\right)$ and $o_{2}=\left(A_{2}, B_{2}, E_{2}\right)$, we would have the following:

"o $0_{1}$ reduces to $o_{2}$ if there is a Chu morphism $(\varphi, \psi)$ witnessing that: whenever $b \in B_{2}$ and $a \in A_{1}, b \in B_{2}$ is a solution for the instance $\varphi(a)$ of $o_{2}$ if, and only if, $\psi(b) \in B_{1}$ is a solution for the instance a of $o_{1}$."

A full investigation on the action and influence of Chu categories on the context of the results of this paper certainly deserves further efforts. However, the application of Chu transforms does not seem particularly promising: for instance, it is easy to see that the morphism of $\mathcal{P} \mathcal{V}$ given by $(\varphi, \varphi)$ in the proof of Proposition 2.1 (which has witnessed the reduction of problems of $\mathcal{C}_{2}$ to problems of $\mathcal{C}_{1}$ ) is not, in general, a Chu morphism.

The following question was raised by Joan Bagaria, during a session of the Barcelona Set Theory Seminar: 
Question 4.4. What happens, in the context of the results of this paper, if we require the constituent functions of all morphisms to be Borel functions?

Indeed, there is a well-known, classical argument of Measure Theory (which is due to Sierpinski and consists of a smart application of Fubini's Theorem) which shows that there are no measurable well-orderings of the real line, and therefore all morphisms defined in terms of well-orderings of $\mathbb{R}$ (either with order type $\mathfrak{c}$ or with order type $\omega_{1}$, depending on the assumptions) are not constituted by Borel functions. In particular, the morphisms which are used in the proofs of Proposition 2.1 and Theorem 2.2 (implication $(i) \Rightarrow(i i)$ ) are not Borel morphisms, so to say. It is surely interesting to wonder what happens if we restrict ourselves to Borel morphisms.

To finish, a disclaimer: the author refrains from going much further on the philosophical discussion of the pure $\mathbf{Z F C}$ theorems presented in this paper. However, he believes that those results will be appealing not only for mathematicians, but for mathematical philosophers as well - since our Main Question (Question 4.1) may raise a number of inquiries and issues on themes such as complexity, probability, randomness and arbitrariness, applicability of reductions between problems, simultaneity and symmetry, etc.; not to mention the inquiries and issues related to the Continuum Hypothesis itself.

\section{Acknowledgements}

The author thanks his colleagues Fabio H. V. Martinez and Thierry C. Petit-Lobão for interesting discussions on the subject of complexity (in computer science, as much as in science in general).

The author also acknowledges his colleagues Hugo Mariano, Odilon Luciano and Francisco Miraglia, from the University of Sao Paulo, for a number of discussions during the Cantor Meetings Robinson Conference (Campinas, Brazil, December 2018) which inspired this work. He also would like to thank Giorgio Venturi for having organized such a lovely conference.

This research was made during a visit of the author to the IMUB, Universitat de Barcelona (from November 2018 till June 2019), and he would like to thank Joan Bagaria for his hospitality. 
Funding. The author was supported by FAPESB, Fundação de Amparo à Pesquisa do Estado da Bahia, Grant APP0072/2016, and CAPES Brazil, Grant PVEX 88881.170714/2018-01.

\section{References}

[1] L.F. Aurichi, Sobre a Hipótese do Contínuo. Algumas Equivalências e Aplicaçoes, MsC Dissertation (in Portuguese), USP University of Sao Paulo, Brazil, 2005.

[2] M. Barr, *-autonomous categories. With an appendix by Po-Hsiang Chu, Lecture Notes in Mathematics 752, Berlin, Heidelberg, New York, Springer-Verlag, 1979, vi $+140 \mathrm{pp}$.

[3] A. Blass, Questions and Answers - A Category Arising in Linear Logic, Complexity Theory, and Set Theory, In: J.-Y. Girard, Y. Lafont, and L. Regnier (eds.), Advances in Linear Logic, London Math. Soc. Lecture Notes 222 (1995), 61-81.

[4] A. Blass, Propositional Connectives and the Set Theory of the Continuum, CWI Quarterly 9 (1996), 25-30.

[5] C. Freiling, Axioms of Symmetry: throwing darts at the real number line, Journal of Symbolic Logic 51:1 (1986), 190-200.

[6] H. Garcia and S.G. da Silva, Identifying small with bounded: unboundedness, domination, ideals and their cardinal invariants, South American Journal of Logic 2:2 (2016), 425-436.

[7] M.R. Garey and D.S. Johnson, Computers and intractability. A guide to the theory of NP-completeness, A Series of Books in the mathematical Sciences, San Francisco, W.H. Freeman and Company, 1979, x +338 pp.

[8] D.G. Green and T. Leishman, Computing and Complexity - Networks, Nature and Virtual Worlds, In: C.A. Hooker, D.M. Gabbay, P. Thagard, J. Woods (eds.), Handbook of the Philosophy of Science, Vol. 10 - Philosophy of Complex Systems, 1st ed., North Holland, 2011, pp. 137-161.

[9] K. Kunen, Set theory. An introduction to independence proofs, Studies in Logic and the Foundations of Mathematics, Vol. 102, Amsterdam, North-Holland Publishing Company, 1980, xvi +313 pp.

[10] P. Maddy, Believing the axioms. I, Journal of Symbolic Logic 53:2 (1988), 481-511.

[11] J.T. Moore, M. Hrušák, and M. Džamonja, Parametrized $\diamond$ principles, Transactions of Americal Mathematical Society 356:6 (2004), 2281-2306.

[12] A. Moreno and M. Mossio, Biological Autonomy: A Philosophical and Theoretical Enquiry, History, Philosophy and Theory of the Life Sciences 12, Dordrecht, Springer, 2015, xxxiv +221 pp.

[13] V. de Paiva, A dialectica-like model of linear logic, In: D. Pitt, D. Rydeheard, P. Dybjer, A. Pitts, and A. Poigne (eds.), Category Theory and Computer Science, Springer, 1989, pp. 341-356. 
[14] V. de Paiva, The Dialectica Categories, Computer Laboratory, University of Cambridge, 1990.

[15] V. de Paiva, Dialectica and Chu constructions: cousins?, Theory and Applications of Categories 17 (2006), 127-152.

[16] C.H. Papadimitriou, Computational complexity, Addison-Wesley Publishing Company, Amsterdam, 1994, xv +523 pp.

[17] S.M. Ross, A first course in probability, 9th ed., Pearson Education/Prentice Hall, Upper Saddle River, NJ, 2014, xi + 467 pp.

[18] W. Sierpiński, Hypothèse du Continu, Monografie Matematyczne, 1ère ed., PWN, Varsóvia, 1934, v + 192 pp.

[19] S.G. da Silva and V. de Paiva, Dialectica categories, cardinalities of the continuum and combinatorics of ideals, Logic Journal of the IGPL 25:4 (2017), 585-603.

[20] S.G. da Silva, The Axiom of Choice and the Partition Principle from Dialectica Categories, 2018. Submitted.

[21] P. Vojtáš, Generalized Galois-Tukey-connections between explicit relations on classical objects of real analysis, In: H. Judah (ed.), Set theory of the reals (Ramat Gan, 1991), Bar-Ilan Univ., Ramat Gan, Israel Math. Conf. Proc. 6, 1993, pp. 619-643.

Instituto de Matemática e Estatística

Universidade Federal da Bahia

Campus de Ondina, Av. Adhemar de Barros, S/N

Ondina, CEP 40170-110, Salvador, BA, Brazil

samuel@ufba.br 